\title{
Performance Evaluation of Load-Balanced Clustering of Wireless Sensor Networks
}

\author{
Gaurav Gupta and Mohamed Younis \\ Dept. of Computer Science and Elec. Eng. \\ University of Maryland Baltimore County \\ Baltimore, MD 21250 \\ \{gagupta1, younis\}@.cs.umbc.edu
}

\begin{abstract}
Wireless sensor networks have received increasing attention in the recent few years. In many military and civil applications of sensor networks, sensors are constrained in onboard energy supply and are left unattended. Energy, size and cost constraints of such sensors limit their communication range. Therefore, they require multi-hop wireless connectivity to forward data on their behalf to a remote command site. In this paper we investigate the performance of an algorithm to network these sensors in to well define clusters with lessenergy-constrained gateway nodes acting as clusterheads, and balance load among these gateways. Load balanced clustering increases the system stability and improves the communication between different nodes in the system. To evaluate the efficiency of our approach we have studied the performance of sensor networks applying various different routing protocols. Simulation results shows that irrespective of the routing protocol used, our approach improves the lifetime of the system.
\end{abstract}

Keywords: Network clustering, Energy-efficient design, Energy-aware communication, Sensor networks.

\section{Introduction}

In recent years, networking of unattended sensing devices has received significant interest from the research community. Sensor networks can increase the efficiency of many military and civil applications, such as combat field surveillance, security and disaster management where conventional approaches prove to be very costly and risky. Sensors are capable to monitor a wide variety of ambient conditions such as: temperature, pressure, motion etc. The sheer number of these devices and their ad-hoc deployment in the area of interest brings numerous challenges in networking and management of these systems. Sensors in such systems are typically disposable and expected to last until their energy drains. Therefore, energy is a very scarce resource for such sensor systems and has to be managed wisely in order to extend the life of the sensors for the duration of a particular mission.

Sensors are generally equipped with data processing and communication capabilities. The sensing circuit measures parameters from the environment surrounding the sensor and transforms them into an electric signal. Processing such a signal reveals some properties about objects located and/or events happening in the vicinity of the sensors. Typically the sensor sends such sensed data, usually via radio transmitter, to 0-7803-7661-7/03/\$17.00C2003 IEEE a base station or Command node, either periodically or based on events. The command node can be statically located in the vicinity of the sensors or it can be mobile so that it can move around the sensors and collect data. In either case, the command node cannot be reached efficiently by all sensors in the system. To avoid long haul communication with the command node some high-energy nodes called "Gateways" are typically deployed in the network. These Gateways, group sensors to form distinct clusters in the system and manage the network in the cluster, perform data fusion to correlate sensor reports and organize sensors by activating a subset relevant to required missions or tasks as shown in Fig 1. Each sensor only belongs to one cluster and communicates with the command node only through the gateway in the cluster.

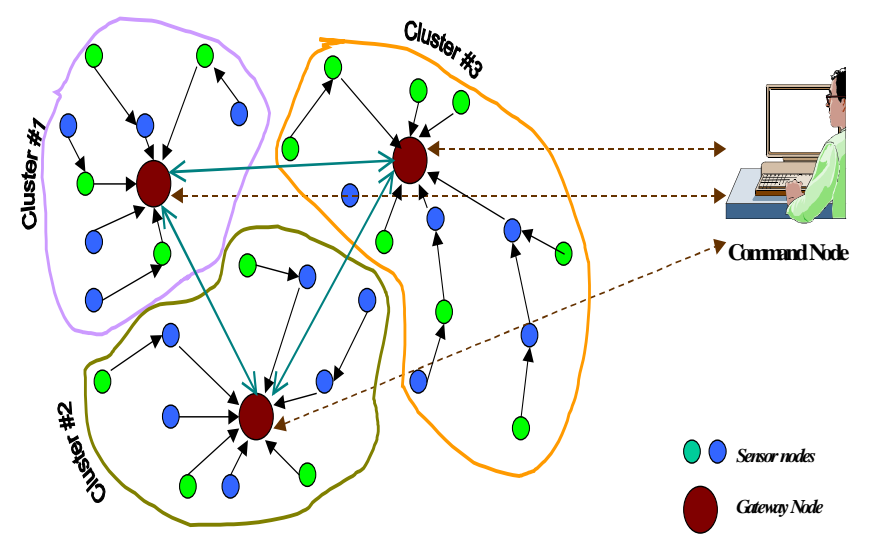

Fig. 1: Multi-gateway clustered sensor network

Signal processing and communication activities are the main consumers of sensor's energy. Since sensors are battery operated, keeping the sensor active all the time will limit the duration that the battery can last. Therefore, optimal organization and management of the sensor network is very crucial in order to perform the desired function with an acceptable level of quality and to maintain sufficient sensors' energy to last for the duration of required missions. Missionoriented organization of the sensor network enables the appropriate selection of only a subset of the sensors to be turned on and thus avoids wasting the energy of sensors that do not have to be involved [6]. In addition, energy-aware routing of communication traffic and medium access arbitration among sensors extend the network lifetime [7].

Similar to other communication networks, scalability is one of the major design's quality attributes for sensor 
networks. A multi-gateway architecture is required to cover a large area of interest without degrading the service of the system. But, if the sensors and gateways are not uniformly distributed then it can cause few gateways to overload with the increase in sensor density, system missions and detected targets/events. Such overload might cause latency in communication and inadequate tracking of targets or events. In order to avoid such patches of dense clusters we balance the load at clustering time to keep the density of the clusters uniform. Simulation results shows that if the load is not balanced among different clusters then few gateways will be heavily loaded after clustering and will consume their energy very soon. This will lead to either all the sensors belonging to that cluster to be inactivated or re-clustering of the system. Frequent reclustering will require extra overhead and change in the system setup. Gateways will have to reschedule the sensors and recalculate the communication paths. Also these additional nodes will add extra burden on all the other gateways.

In the next two sections we define the architectural model of sensor network and summarize related work. In Section 4 we explain the algorithm for balanced clustering of a sensor network. Description of the simulation environment, analysis of the experimental results and comparison with other routing approaches can be found in section 5. Finally section 6 concludes the paper and discusses our future research plan.

\section{System Model}

The system architecture for the sensor network is shown in Fig. 1. Gateway nodes are less-energy-constrained compared to sensors. The sensors and gateways are assumed to be of the same kind and have same properties respectively. All communication is over wireless links. A wireless link is established between two nodes only if they are in range of each other. Links between two sensors are considered bidirectional while links between a gateway and sensor can be unidirectional depending on the range of the sensor. Gateways are capable of long-haul communication compared to the sensors and all gateways are assumed to be in communication range with one another. Communication between nodes is over a single shared channel. Current implementation supports TDMA [7] protocol to provide MAC layer communication.

In this paper we assume that the sensor and gateway nodes are stationary. In the future we plan to incorporate mobile gateways in the system. During the bootstrapping process, all the sensors and gateways are assigned unique IDs, initial energy and TDMA schedule. The TDMA schedule is only valid for the first phase of the clustering, after that the assigned gateways provide new schedule to the nodes in their clusters. The sensor is assumed to be capable of operating in an active mode or a low-power stand-by mode. The sensing and processing circuits can be powered on and off. In addition both the radio transmitter and receiver can be independently turned on and off and the transmission power can be programmed based on the required range. It is also assumed that the sensor can act as a relay to forward data from another sensor. The on-board clocks of both the sensors and gateways are assumed to be synchronized, e.g. via the use of GPS. Also, all nodes are assumed to be aware of their position through some GPS system. While the GPS consumes significant energy, it has to be turned on for a very short duration during bootstrapping. Sensors inform the gateways about their location during the clustering process. It is worth noting that most of these capabilities are available on some of the advanced sensors, e.g. the Acoustic Ballistic Module from SenTech Inc. [2]

\subsection{Sensor's Energy Model}

A typical sensor node consists mainly of a sensing circuit for signal conditioning and conversion, digital signal processor, and radio links $[3,4]$. The following summarizes the energyconsumption models for each sensor component.

Communication Energy Dissipation: The key energy parameters for communication in this model are the energy/bit consumed by the transmitter electronics $\left(\alpha_{t}\right)$, energy dissipated in the transmit op-amp $\left(\alpha_{\text {amp }}\right)$, and energy/bit consumed by the receiver electronics $\left(\alpha_{\mathrm{r}}\right)$. Assuming a $1 / \mathrm{d}^{2}$ path loss, the energy consumed is:

$$
E_{t x}=\left(\alpha_{t}+\alpha_{a m p} d^{2}\right) * r \text { and } E_{r x}=\alpha_{r} * r
$$

Where $E_{t x}$ is the energy to send $r$ bits and $E_{r x}$ is the energy consumed to receive $r$ bits. Table 1 summarizes the meaning of each term and its typical value.

\begin{tabular}{|c|l|}
\hline erm & \multicolumn{1}{|c|}{ Meaning } \\
\hline$\alpha_{t}, \alpha_{r}$ & $\begin{array}{l}\text { Energy dissipated in transmitter and receiver } \\
\text { electronics per bit (Taken to be } 50 \mathrm{~nJ} / \mathrm{bit}) .\end{array}$ \\
\hline$\alpha_{a m p}$ & $\begin{array}{l}\text { Energy dissipated in transmitter amplifier } \\
\left(\text { Taken }=100 \mathrm{pJ} / \mathrm{bit} / \mathrm{m}^{2}\right) .\end{array}$ \\
\hline$r$ & Number of bits in the message. \\
\hline$d$ & Distance that the message traverses. \\
\hline
\end{tabular}

Table 1: Parameters for the communication energy

Computation Energy Dissipation: We assume the leakage current model of $[4,8]$. The model depends on the total capacitance switched and the number of cycles the program takes. We used parameter values similar to those in [8].

\section{Related Work}

Our work is motivated by a various research projects in sensor network domain. Researchers are exploring both hardware and software aspect of sensor networks. Projects like Smartdust [9], WINS [10], PicoRadio [11] have given a new prospective to the size and capabilities of sensors. Since sensors are typically battery-operated with limited energy supply, many research groups have focused on issues like energy aware routing [12], sensor coordination [13], and energy saving through activation of a limited subset of nodes [14].

Clustering of nodes in wireless network is a wellresearched field. Most of the published approaches for clustering base the selection of a cluster-head on different 
factors like cluster ID [16], degree of connectivity $[17,18]$ or randomized [15]. Frequent selection of clusterheads is desired if the topology is constantly changing or the load has to be shared among all the nodes. If traditional clustering approaches like lowest/highest ID or highest connectivity are applied, the same node will be picked as cluster-head every time, resulting in this sensor to drain its energy very fast. Obviously picking a resource-constrained sensor node for acting as a processing element and a relay for the cluster will quickly deplete its energy. Rotation of the role of the clusterhead among other sensors in the cluster, based on a roundrobin strategy [15], node connectivity or battery energy [17] changes the topology of clusters frequently. Cluster-head change imposes huge overhead since all other cluster-heads have to be notified about the change.

Moreover published clustering protocols do not consider any balancing of load among clusters due to variable density of nodes in the system. If load is not balanced in the system there can be clusters with high-density and very low-density. In such scenarios the high-density cluster-head will be overwhelmed with processing and communication load and will consume its energy soon, while the low density clusterhead will sit idle wasting precious time. Our algorithm balances the load among the clusters to increase the system lifetime and improves the performance of different routing algorithms.

\section{Load-Balanced Clustering}

The main objective of our approach is to cluster sensor network efficiently around few high-energy gateway nodes. Clustering enables network scalability to large number of sensors and extends the life of the network by allowing the sensors to conserve energy through communication with closer nodes and by balancing the load among the gateway nodes. Gateways associate cost to communicate with each sensor in the network. Clusters are formed based on the cost of communication and the load on the gateways.

Network setup is performed in two stages; 'Bootstrapping' and 'Clustering'. In the bootstrapping phase, gateways discover the nodes that are located within their communication range. Gateways broadcast a message indicating the start of clustering. We assume that receivers of sensors are open throughout the clustering process. Each gateway starts the clustering at a different instance of time in order to avoid collisions. In reply the sensors also broadcast a message with their maximum transmission power indicating their location and energy reserve in this message. Each node discovered in this phase is included in a per-gateway range set.

In the clustering phase, gateways calculate the cost of communication with each node in the range set. This information is then exchanged between all the gateways. After receiving the data from all other gateways each gateway start clustering the nodes based on the communication cost and the current load on its cluster. When the clustering is over, all the sensors are informed about the ID of the cluster they belong to. Since gateways share the common information during clustering, each sensor is picked by only one gateway. For inter-cluster communication all the traffic is routed through the gateways.

\subsection{Problem Formulation}

Each gateway constructs a range set of all the nodes that can communicate with it. A sensor ' $\mathrm{S}_{j}$ ' belongs to range set 'RSet' of gateway ' $\mathrm{G}_{\mathrm{i}}$ ' if it satisfies the following criteria:

$$
S_{j} \in \operatorname{Ret}_{G i} \Leftrightarrow\left[\left(R_{G i}>d_{S j->G i}\right) \Lambda\left(R_{S j, \max }>d_{S j-->G i}\right)\right]
$$

Where, $R_{G i}$ is the range of gateway $G_{i}, R_{S j, \max }$ is the maximum range of sensor $S_{j}$ and $d_{S j->G i}$ is the distance between sensor $S_{j}$ and Gateway $G_{i}$. Each node in the range set is associated with a communication cost calculated by the gateway. The cost calculated is a function of communication energy dissipated in transferring ' $r$ ' bits of data over a $d_{S j>G i}$ distance. Using the energy model described in section 2.1, the cost ' $C_{j, G i}$ ' associated with sensor $S_{j}$, calculated by gateway $G_{i}$ is:

$$
C_{j, G i}=E_{t x}+E_{r x}=\left(\alpha_{t}+\alpha_{a m p}\left(d_{S j->G i}\right)^{2}\right) * r+\alpha_{r} * r
$$

A per cluster record is created with all the nodes in RSet and their associated cost. The record is exchanged by all the gateways to gain global knowledge of the network. Depending on the range of the sensors there can be two kinds of nodes in the system: the first can only communicate with one gateway, 'exclusive nodes' and the second can communicate with more then one gateway. The reach of a node is defined as the number of gateways it can communicate with. The first step towards clustering is to separate exclusive nodes from the rest, because these are compulsory nodes to be accommodated by a gateway. To do so, gateways construct another set called exclusive set 'ESet' which consists of nodes that can satisfy the following criteria:

$$
S_{j} \in \operatorname{ESet}_{G i} \Leftrightarrow\left[\left(S_{j} \in \operatorname{RSet}_{G i}\right) \Lambda\left(\forall k \neq i, S_{j} \notin \operatorname{RSet}_{G k}\right)\right]
$$

The load on a gateway is a function of processing load ' $P L_{G i}$ ' and communication load ' $C E_{G i}$ ' due to sensors in the cluster and is defined as:

$$
L_{G i}=f\left(P L_{G i}, C E_{G i}\right)
$$

Processing load on a gateway is due to processing the data from the sensors in the cluster and energy consumed in doing so. Communication energy, ' $C E_{G i}$ ' of a gateway is calculated to be the summation of the communication cost of all sensors in the cluster. That is,

$$
C E_{G i}=\sum_{\mathrm{j}=0}^{\mathrm{n}} \mathrm{C}_{\mathrm{j}, \mathrm{i}}
$$

Since, we assume that all sensors are identical and produce data at the same rate, $P L_{G i}$ of a gateway is directly proportional to the number of sensors ' $n$ ' in the cluster. It implies that, to balance load in the system we have to balance the number of nodes in a cluster and the communication energy required per gateway.

In order to keep the system close to the average load, we choose an objective function that minimizes the variance of the cardinality of each cluster in the system. That is, 


$$
\sigma^{2}=\frac{1}{G} \sum_{\mathrm{i}=0}^{\mathrm{G}}\left(\mathrm{X}-\mathrm{X}^{\prime}\right)
$$

Where ' $\sigma^{2}$ ' is the variance of load in the system, $\mathrm{X}$ is cardinality of gateway $G_{i}$ and $X$ ' is the average cardinality including the node under consideration, $G$ is the total number of gateways in the system.

\subsection{Optimization Heuristics}

Before minimizing the objective function we allocate the nodes in the ESet to their respective clusters and calculate the load. If we allocate the remaining nodes to the clusters only by minimizing the objective function we experience large overlapping of clusters. Considering only the load on gateways as a factor for clustering might do so at the expense of sensors. Our experiments also show that some sensors are not part of the gateways nearest to them. This will increase the communication energy of the sensors.

Exhaustive search methods like simulated annealing can be used to find the optimum results to balance the load as well as maintain the minimum distance with the gateway. But by using these methods the complexity of the algorithm is increased with the increase in sensors and gateways. In order to balance load of gateways and preserve precious energy of sensors, we select few nodes that are located radically near a gateway and include them in the ESet of the gateway. A node is included in the ESet of a gateway if, its distance to the gateway is less then a critical distance. Initially, the critical distance is equal to the minimum distance in the ESet. Then the critical distance is gradually increased till the median of distances in ESet is reached. This procedure is repeated for all the gateways based on increasing order of cardinality.

Now, we start clustering the remaining sensors in the system. Since sensors cannot reach all the gateways, minimizing objective function for those gateways will unnecessarily increase the complexity of algorithm. In order to save computation for clustering we sort the sensors based on increasing order their reach. Nodes with same reach are grouped together to avoid extra computation of calculating the objective function for the gateways they cannot reach. Nodes with lower reach are considered first because they have fewer clusters to join. The objective function is calculated by assigning these nodes to the gateways they can reach. The node becomes the part of the cluster for which it minimizes the objective function. The process is repeated till all the nodes in the sensors are not clustered.

\section{Experimental Evaluation}

To evaluate the effectiveness of our clustering approach we perform simulations to compare the performance of different routing approaches with our load-balanced clustering and shortest distance clustering. This section describes simulation environment, performance metrics and experimental results from the simulations.

\subsection{Environmental Setup}

Experiments are performed on simulations with 100 sensors uniformly distributed in a $1000 \times 1000$ square meter area. Each sensor is assumed to have an initial energy of 0.5 joules and a buffer for up to 15 packets. A node is considered nonfunctional if its energy level reaches 0 joules. The maximum range of the sensors is set to $200 \mathrm{~m}$. Range of the gateways is considered enough to cover the whole area under consideration. It is assumed that the channel is collision free. Packet lengths are fixed to $10 \mathrm{Kbit}$ for data packets and $2 \mathrm{Kbit}$ for routing and refresh packets [12]. Sensors are given IDs in random fashion. Sensors are informed about the first TDMA schedules by their respective gateways. The nodes in a cluster can be in one of four main states: sensing only, relaying only, sensing-relaying, and inactive. In the sensing state, the node sensing circuitry is on and it sends data to the gateway in a constant rate. In the relaying state, the node does not sense the target but its communications circuitry is on to relay the data from other active nodes. When a node is both sensing the target and relaying messages from other nodes, it is considered in the sensing-relaying state. Otherwise, the node is considered inactive and can turn off its sensing and communication circuitry. Gateways can change the state of the sensors based on the routing approach used.

A node sensing a target produces data packets at a constant rate of 1 packet/sec [12]. Each data packet is timestamped when generated to allow tracking delays. In addition, each packet has an energy field that is updated during the packet transmission to calculate energy per packet. A packet drop probability is taken equal to 0.01 . This is used to make the simulator more realistic and to simulate the deviation of the gateway energy model from the actual energy.

We assume that the cluster is tasked with a target-tracking mission. The initial set of sensing nodes is chosen to be the nodes on the convex hull. The set of sensing nodes changes as the target moves. Since targets are assumed to come from outside the cluster, the sensing circuitry of all boundary nodes is always turned on. The sensing circuitry of other nodes are usually turned off but can be turned on according to the target movement. Targets are assumed to start at a random position outside the convex hull. We experimented with different types of targets but for this paper we choose the linearly moving targets. These targets are characterized by having a constant speed chosen uniformly from the range 4 meters/s to 6 meters/s and a constant direction chosen uniformly depending on the initial target position when crossing the convex hull.

\subsection{Performance Metrics}

We used the following metrics to capture the performance of different routing algorithms.

- Time to network partition: When the first node runs out of energy, the network within a cluster is said to be partitioned $[5,12]$, reflecting the fact that some routes become invalid.

- Average Lifetime of sensors: This metric, along with the time to network partition, gives an indication of network lifetime. 
- Average delay per packet: Defined as the average time a packet takes from a sensor node to the gateway. Although energy is an important factor in sensor networks, some mission critical applications requires sensed data to be reported with minimum delay.

- Network Throughput: Defined as total number of packets received at the gateway divided by the simulation time. This metric gives a good idea of the efficiency of network traffic supported by each cluster. A high throughput indicates that the system supports better routing for data and control messages.

- Average energy consumed per packet: Minimizing the energy per packet will, in general, yield better energy savings.

- Average Power Consumed: This metric is an average of power consumed taken at different instance of time during the simulation. It indicates the power utilized due to message traffic in the system.

- Standard Deviation of Load per cluster: To test our system for different sensors densities we measured standard deviation of load by using 5 gateways and increasing the number of sensors in the system from 100 to 500 with uniform increments.

\subsection{Performance Results}

In this section we present the results obtained from our simulations. We compare the performance metric described above for different routing algorithms in clusters formed by our load-balanced clustering approach and shortest distance clustering approach. In shortest distance clustering approach all the sensors in the system are sorted based on the distance with each cluster. A sensor joins the cluster with the shortest distance between the sensor and the gateway. To demonstrate the effect of load-balancing in clusters we integrated our clustering with six different well-known routing approaches. Fig 2-7 demonstrates the results obtained for the following routing approaches:

DEnergy-Aware Routing: Sensors messages are routed through multiple hops based on the current energy level of the sensors, distance, delay, in-out traffic, etc. [12].

> Minimum-Hop Routing: A packet is forwarded to the gateway using the minimum number of hops in the cluster.

$>$ Direct Routing: Messages are directly transmitted to the gateways. No intermediate hops or routes are created in the system.

$>$ Minimum-Distance Routing: This approach favors the use of a closer node as a hop, leading to a longer end-to end delay.

> Minimum-Distance Square Routing: Routes are set favoring closer hops, with the objective of minimizing the sum of the square of inter-hop distance.

$>$ Linear-Battery Routing: Minimize energy consumption assuming linear battery discharge model [5].

Comparison between routing algorithms:

One of the main objectives of our approach is to increase the stability of the system. When a node dies new routes have to be recalculated and routing tables are updated in the whole network. Figures 2 and 3 indicate the improvement in the network partitioning time and average lifetime of the sensors in the system. Fig. 2 shows that we increase the network partition time by a factor of 4 and 2 for energy aware routing and minimum hops routing. We are better then shortest distance clustering for minimum distance square routing by a factor of 7 because the minimum distance routing will try to find the shortest path which will be through a set of few nodes located very near the gateway. Since the clusters are not balanced in some clusters those nodes will be overwhelmed with relay traffic and will die very soon.

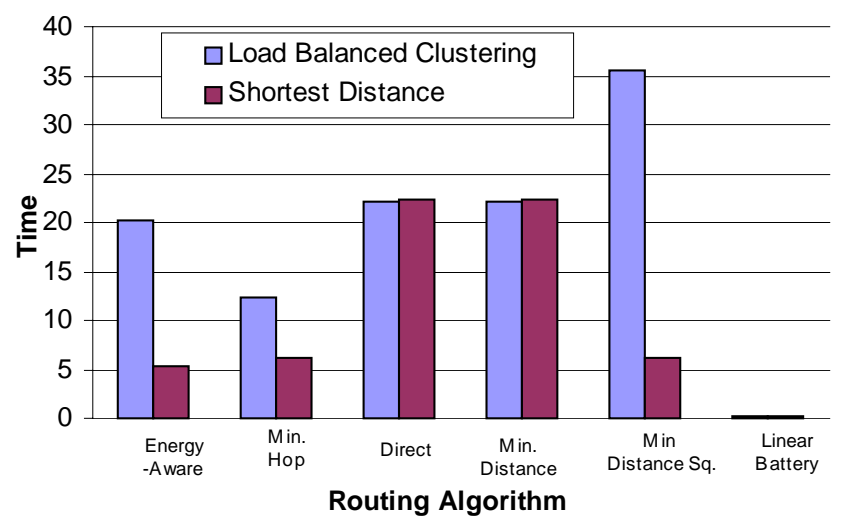

Fig. 2: Time for first node to die (Network Partitioning)

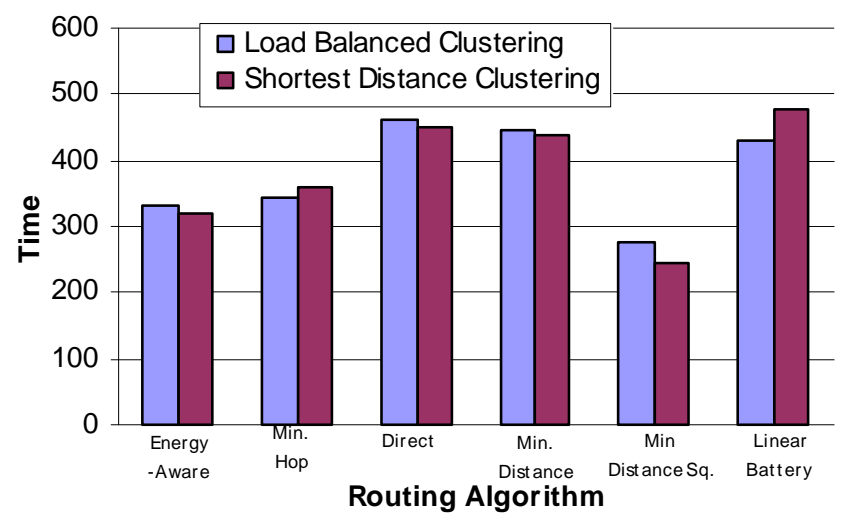

Fig. 3: Average Lifetime of the sensors

Fig. 3 demonstrate that in all algorithm the average lifetime of the sensors are almost equal because in shortest distance clusters nodes will die quickly in the beginning as shown in Fig. 2 and it is obvious that shortest distance clustering will perform better in sparse networks therefore the system lifetime will be equivalent to the load-balanced clustering. However, the large number of sensors loss in the beginning affects the overall throughput of the system (Fig. 4).

Fig. 5 shows the average delay per packet in the system. Packet delay is an important metric to evaluate the efficiency of a network. Many mission critical applications like disaster management and target tracking requires packets to be delivered to the destination as soon as any significant event occurs. Unbalanced clusters create additional delays in delivering sensed data packets. Results demonstrate that 


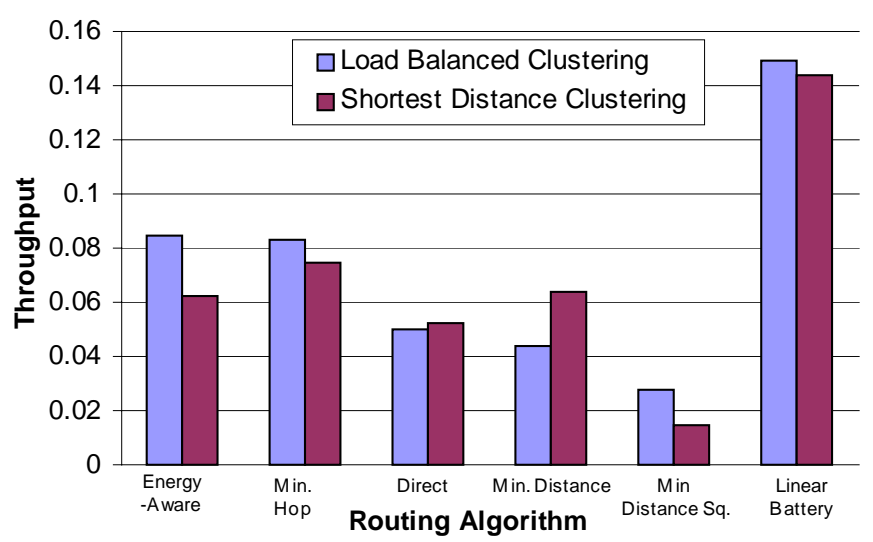

Fig. 4: Throughput of the System

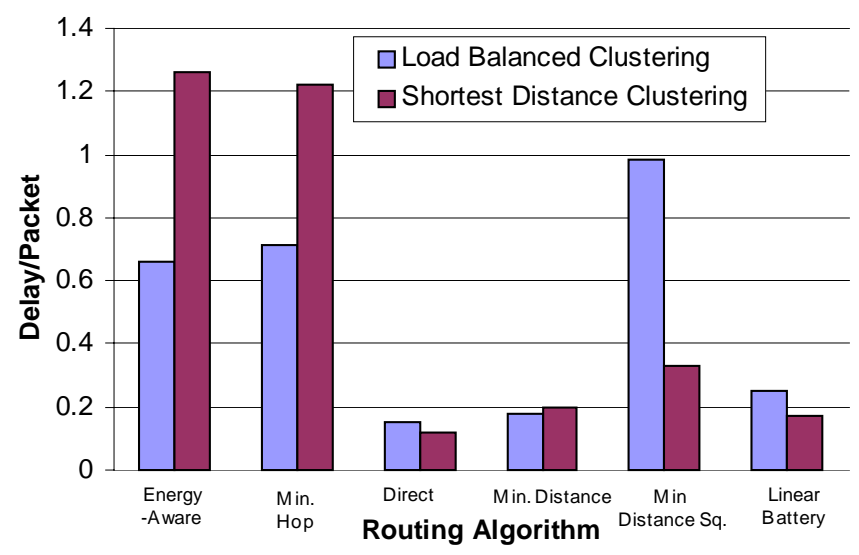

Fig. 5: Average Delay per Packet

networks using our approach and energy-aware or minimum hops routing produces delays equal to half the delay as compared to shortest distance clusters. Only minimum distance square routing using shortest distance clustering performs better then our algorithm, however such routing approach performs very bad in terms of time to network partitioning (Fig. 2). For rest of the routing approaches packet delay is almost the same for both the clustering approaches.

We calculated the average energy consumed per packet at the end of the simulations and compared the results. Linear battery routing strictly performs the routing based on the battery power of the sensors therefore the energy consumed per packet is same for both the approached as shown in Fig. 6 . Although direct, min distance and min distance square performs by an average 1.17 times better then our approach we outperform shortest distance clustering by a factor of 1.24 in energy-aware and minimum hops routing.

Results shown in Fig. 7 clarify the energy consumption in the system due to message exchange. The power consumed in the system was calculated at different instance of times for each cluster and an average is taken. As the figure shows we conserve more power in almost all the cases by a factor of more then 1.5 then the other approach. Such saving is due to reduction in packets drops, delays and system overload caused in overloaded clusters.

Fig 8 presents the stability of the clusters under different load conditions. The standard deviation of load indicates that



Fig 6. Average Energy per Packet

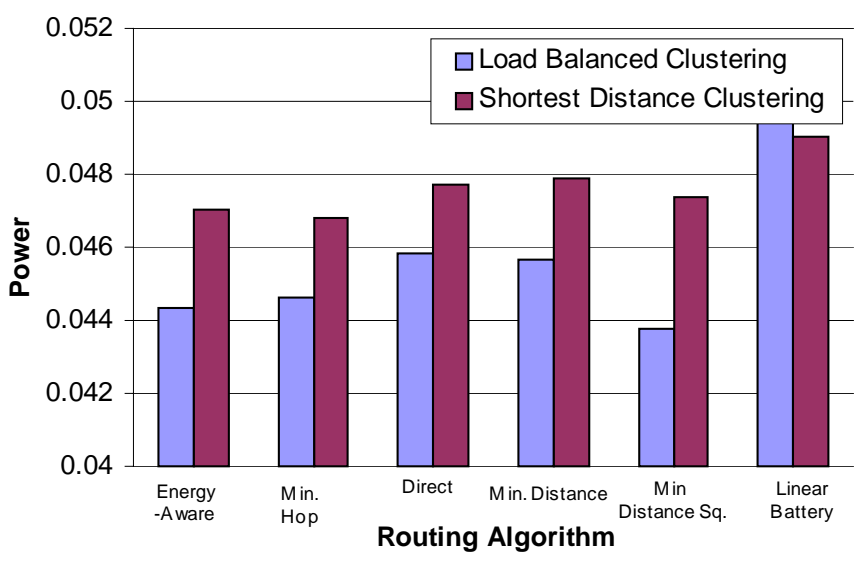

Fig 7. Average power consumed in communication

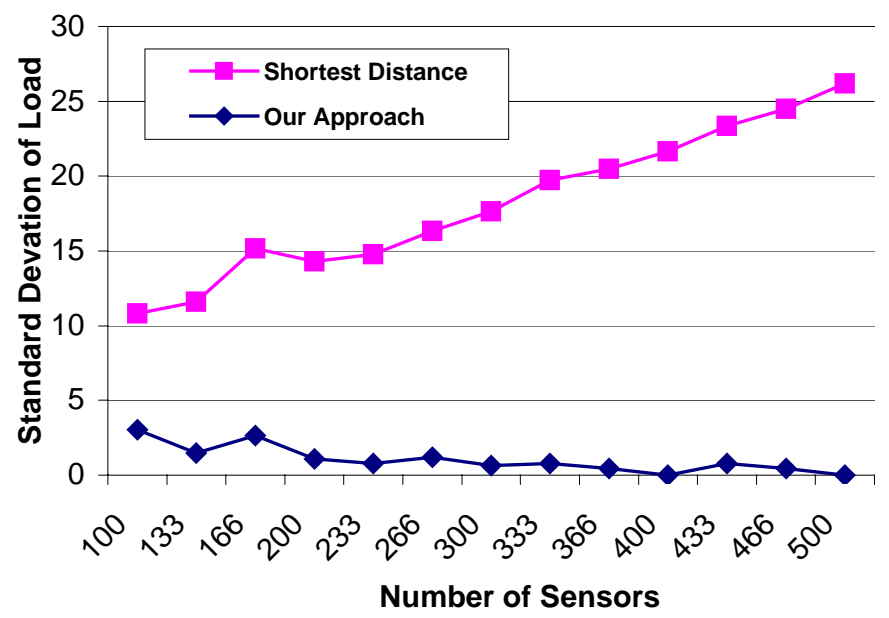

Fig 8. Standard Deviation of Load with increase in sensor's density

the performance of our approach is constant with increase in density. On the other hand the rising curve of the shortest distance clustering clearly indicates that the variance of load among different clusters increases, as more sensors are included in the system. The demonstrated results are based on the normal distribution of sensors. 


\section{Conclusion}

In this paper we investigated the performance of a new clustering algorithm for sensor networks. The proposed approach balances the load among clusters and simultaneously tries to cluster the sensor nodes as close to high-energy cluster-heads, called gateways. Simulation results have demonstrated the efficiency of load balanced clustering for sensor networks applying different routing methodologies. We covered a gamut of performance parameters for six different routing approaches and demonstrate that our approach improves most of the metrics important for wireless sensor networks.

We plan to extend the clustering in future for mobile sensors and gateways and compare the results with different ad-hoc clustering approaches. We also plan to include faulttolerance in the system by performing recovery from gateway failures.

\section{References}

[1] R. Burne, et. al, "A Self-Organizing, Cooperative UGS Network for Target Tracking," Proc. of SPIE Conference on Unattended Ground Sensor Tech. and Applications II, Orlando, April 2000.

[2] "Data sheet for the Acoustic Ballistic Module", SenTech Inc., http://www.sentech-acoustic.com/

[3] M. Bhardwaj, et. al, "Upper Bounds on the Lifetime of Sensor Networks", In Proceedings of ICC 2001, June 2001.

[4] R. Min, et. al, "An Architecture for a Power-Aware Distributed Microsensor Node", IEEE Workshop on Signal Processing Systems (SiPS '00), October 2000.

[5] S. Singh, M. Woo and C. S. Raghavendra, "Power-Aware Routing in Mobile Ad Hoc Networks", Proc. of ACM MOBICOM'98, Dallas, Texas, October 1998

[6] B. Chen, et al., "Span: An Energy-Efficient Coordination Algorithm for Topology Maintenance in Ad Hoc Wireless Networks", Proc. of MobiCom 2001, Rome, Italy, July 2001.

[7] K. Arisha, M. Youssef, M. Younis, "Energy-Aware TDMABased MAC for Sensor Networks," IEEE Workshop on Integrated Management of Power Aware Communications, Computing and Networking (IMPACCT 2002), May 2002.

[8] A. Sinha and A. Chandrakasan, "Energy Aware Software", Proceedings of the 13th International Conference on VLSI Design, pp. 50-55, Calcutta, India. January 2000.

[9] J.M. Kahn, R.H. Katz, K.S.J. Pister, Next century challenges: Mobile networking for 'smart dust', Proc. MOBICOM, Seattle, 1999

[10] Burnstein, A., Bult, K., Chang, D, Chang, F. et al. "Wireless Integrated Microsensors"; Proceedings Sensors EXPO 1996, Anaheim, CA..

[11] J. Rabaey, J. Ammer, J.L. da Silva, D. Patel, "PicoRadio: Adhoc wireless networking of ubiquitous low-energy sensor/monitor nodes," IEEE Computer Society Workshop on VLSI 2000, Orlando, FL, pp. 9--12, April 2000.

[12] M. Younis, M. Youssef, K. Arisha, "Energy-Aware Routing in Cluster-Based Sensor Networks", in the Proceedings of the $10^{\text {th }}$ IEEE/ACM International Symposium on Modeling, Analysis and Simulation of Computer and Telecommunication Systems (MASCOTS2002), Fort Worth, Texas, October 2002. (To appear)

[13] D. Estrin, R. Govindan, J. Heidemann, and S. Kumar. Scalable coordination in sensor networks. Proc. of ACM/IEEE MobiCom 1999, Seattle, Washington, August 1999
[14] A. Cerpa and D. Estrin, "ASCENT: Adaptive Self-Configuring Sensor Networks Topologies," Proc. INFOCOM 2002, New York, June 2002.

[15] W. Rabiner Heinzelman, A. Chandrakasan, and $\mathrm{H}$. Balakrishnan, "Energy-Efficient Communication Protocols for Wireless Microsensor Networks," Hawaii International Conference on System Sciences (HICSS '00), January 2000.

[16] D.J Baker and A. Ephremides, "A Distributed algorithm for Organizing Mobile Radio Telecommunication Networks", in the Proceedings of the $2^{\text {nd }}$ International Conference in Distributed Computer Systems, April 1981.

[17] M. Gerla and J.T.C Tsai, "Multicluster, mobile, multimedia radio network," ACM/Baltzer Journal of Wireless networks, Vol. 1, No. 3, pp. 255-265, 1995.

[18] A.K. Parekh, "Selecting Routers in Ad-Hoc Wireless Networks", Proceedings of the SBT/IEEE International Telecommunications Symposium, August 1994

[19] F. Zhan, C. Noon, "Shortest Path Algorithms: An Evaluation Using Real Road Networks," Transportation Science, 1996.

[20] C-K. Toh, "Maximum Battery Life Routing to Support Ubiquitous Mobile Computing in Wireless Ad Hoc Networks," IEEE Communications Magazine, June 2001 